Char act eri stics of aer odynamic sound sources gener at ed by coil ed wires in a uni for $m$ ai $r$ - $f$ I ow

\begin{tabular}{|l|l|}
\hline 著者 & $\begin{array}{l}\text { MATSUMTO Hi roki , N SH DA Kohshi, SAI TO } \\
\text { Ken- } \mathrm{i} \text { chi }\end{array}$ \\
\hline $\begin{array}{l}\text { j our nal or } \\
\text { publ i cat i on ti t l e }\end{array}$ & Journal of fl ui ds and st r uct ures \\
\hline vol une & 18 \\
\hline number & 6 \\
\hline page range & $759-770$ \\
\hline year & $2003-12$ \\
\hline URL & ht t p: //hdl . handl e. net /10258/671 \\
\hline
\end{tabular}


Char act eri stics of aer odynamic sound sources gener at ed by coil ed wires in a uni for $m$ ai $r$ - $f$ I ow

\begin{tabular}{|l|l|}
\hline 著者 & $\begin{array}{l}\text { MATSUMTO Hi roki , N SH DA Kohshi, SAI TO } \\
\text { Ken- } \mathrm{i} \text { chi }\end{array}$ \\
\hline $\begin{array}{l}\text { j our nal or } \\
\text { publ i cat i on ti t l e }\end{array}$ & Journal of fl ui ds and st r uct ures \\
\hline vol une & 18 \\
\hline number & 6 \\
\hline page range & $759-770$ \\
\hline year & $2003-12$ \\
\hline URL & ht t p: //hdl . handl e. net /10258/671 \\
\hline
\end{tabular}




\title{
Characteristics of aerodynamic sound sources generated by coiled wires in a uniform air flow
}

\author{
H. Matsumoto*, K. Nishida, K. Saitoh \\ Department of Mechanical Systems Engineering, Muroran Institute of Technology \\ 27-1 Mizumoto-Cho Muroran-City, Hokkaido, 050-8585, Japan
}

\begin{abstract}
This study deals experimentally with aerodynamic sounds generated by coiled wires in a uniform air flow. The heating element of a hair dryer is used as the model. In the experiment, the characteristics of the aerodynamic sound are investigated with relations to the coil diameter $D$, the wire diameter $d$ and the coil spacing $s$. The results showed that an aeolian sound generates when $s / d$ ratio is larger than 1 . Also the peak frequencies of aeolian sounds were higher than that of a straight cylinder having the same diameter $d$. To clarify the characteristics of the aerodynamic sound sources, the directivity of the aerodynamic sound generated by the coiled wire was measured and the coherent function of the velocity fluctuations around the coiled wire and the sound was obtained. Moreover, the band overall value of coherent output power of the sound and the velocity fluctuation have been calculated. This method has clarified the sound source region of the aeolian sound generated by the coiled wire. The current study prevails that the sound source region of the aeolian sound generates by the arc part of the coiled wire, which is located in the upper side of the air flow.
\end{abstract}

\section{Introduction}

The sound environment in our daily life has become uncomfortable due to the many kinds of noises. For example, in the case of a hair dryer, aerodynamic noises and motor noise etc. are generated. If the mechanism of these noise generations is clarified, it becomes possible to take effective counterplans for decreasing such noises. In particular, it is difficult to find the aerodynamic noise source regions. In the studies of this kind done so far, the straight round bar has been mainly chosen as the subject of each study. ( e.g., Fujita et al. (1996,1997), Iida et al. (1998), Mochizuki et al. (1994) ) However, in the practical structures, not only straight round bars but also bent round bars are widely used.

In this study, we focus on the aerodynamic sound generated by the coiled wire. The heating element of a hair dryer is used as the model for the current study. The purpose of this research is to clarify the characteristics of the aerodynamic sound generated by the coiled wire, and to find the wake region that has strong correlation with the aeolian sound by examining the coherence function of the aeolian sound and the velocity fluctuation of the wake behind the coiled wire.

\footnotetext{
*Corresponding author.

E-mail address: h_matsu@mmm.muroran-it.ac.jp (H. Matsumoto).
} 


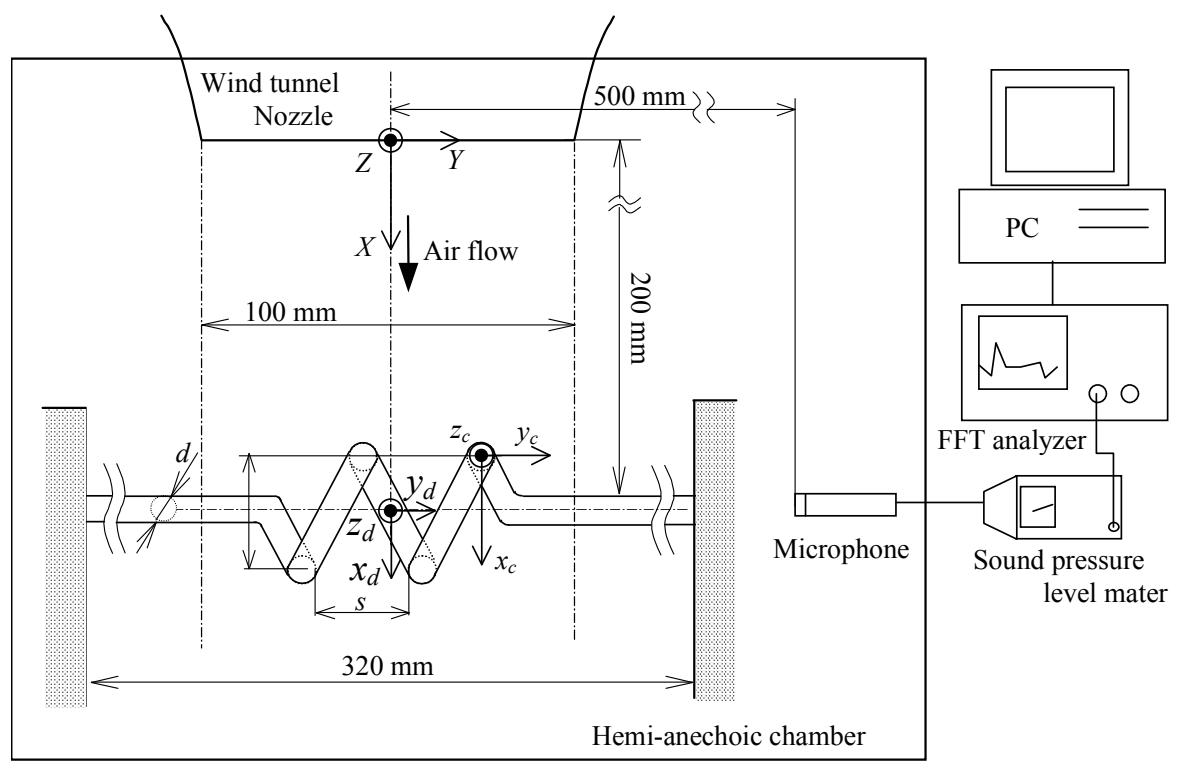

Fig.1 Experimental equipment.

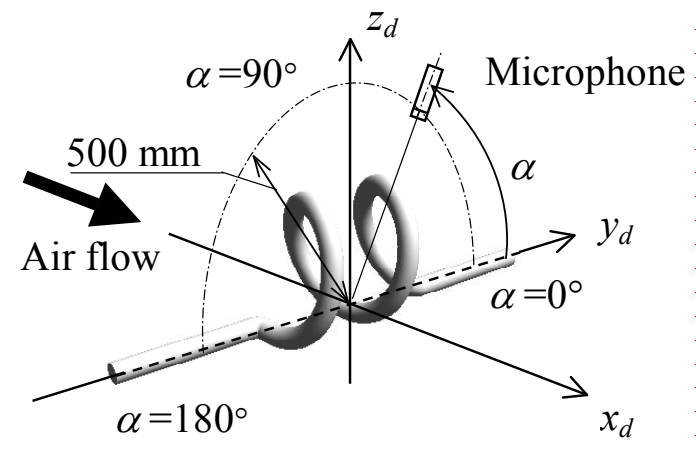

Fig.2 Definition of coordinates for measurement of directivity patterns.

\section{Experimental equipment}

The experimental equipment is shown in Fig.1. The wind tunnel is an open type with a rectangular nozzle of $100 \mathrm{x}$ $100 \mathrm{~mm}$. A precision sound level meter and a hot-wire anemometer are used to measure the aerodynamic sound and the velocity fluctuation, respectively. The air flow velocity is determined by the static pressure differential between the inlet and outlet of the nozzle. The flow in the test-section has a turbulence intensity of $0.8 \%$ in the section of $70 \times 70 \mathrm{~mm}$ at $X=200 \mathrm{~mm}$. The velocity profile of having more than $95 \%$ of uniformity is obtained at the test-section of $80 x 80 \mathrm{~mm}$. Fig. 2 shows the coordinate system used for the current analysis. The microphone is installed in the $y_{d}-z_{d}$ plane at the position of $X=200 \mathrm{~mm}$. The angular position of microphone is indicated by an angle $\alpha$. The tungsten single sensor hot wire probe $(5 \mu \mathrm{m}$ Dia. $1 \mathrm{~mm}$ long) is attached to the traverse device is used to measure the velocity fluctuation at an arbitrary location in the wake behind the coiled wire. All the experiments are performed in a hemi-anechoic chamber. In Fig. $1, D$ is coil diameter, $d$ is wire diameter and $s$ is coil spacing. Total number of turns is 2. In the experiment, the effects of $D, d$ and $s$ on the aerodynamic sound are investigated.

The coiled wire is set up in the horizontal plane containing the center of the nozzle section. Moreover, the axis of coil is located at the position of $X=200 \mathrm{~mm}$ and parallels the $Y$-axis. The wire diameter $d$ is selected as 3 and $4 \mathrm{~mm}$. The mean velocity of air flow, $U$ used is between $20 \mathrm{~m} / \mathrm{s}$ and $40 \mathrm{~m} / \mathrm{s}$. 


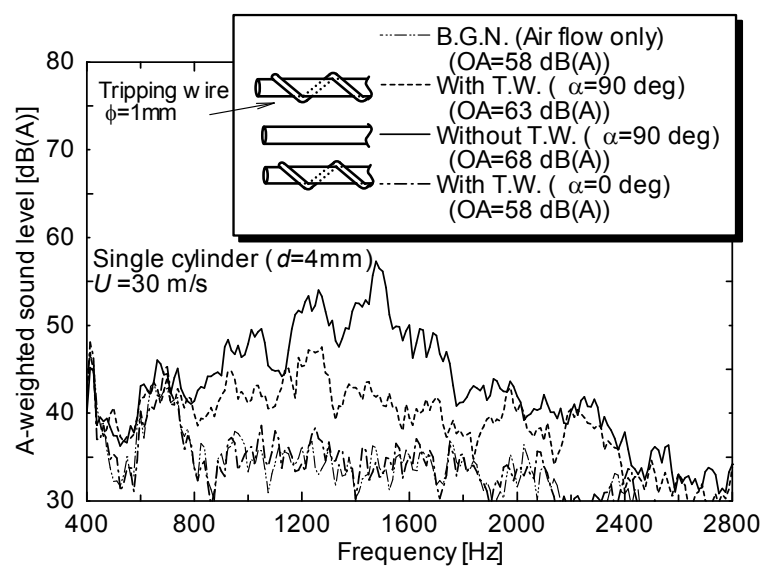

Fig.3 Effect of tripping wire on spectra of aerodynamic sounds generated by cylinders. $(U=30 \mathrm{~m} / \mathrm{s}, d=4 \mathrm{~mm})$

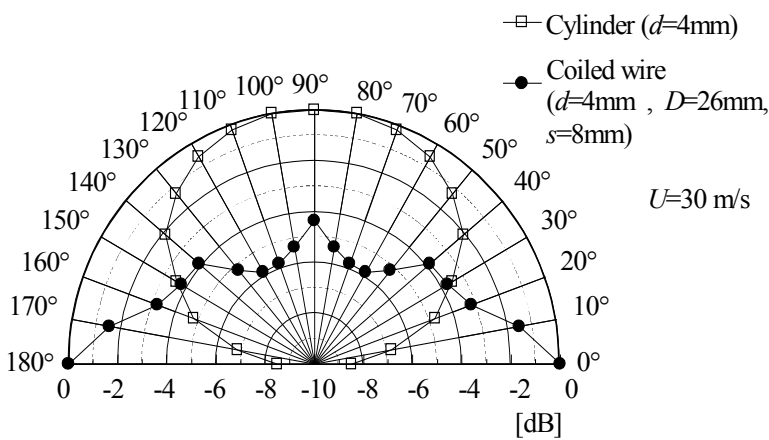

Fig.4 Directivity patterns of aerodynamic sounds generated by the coiled wire and the single cylinder.

\section{Measurement method}

\subsection{Background Noise}

In this study, background noise (BGN) is defined as the noise generated by the air flow. But the coiled wire model has a helical coil part and straight cylinder parts. Thus, the aerodynamic sound generated by this model contains the sound generated by the helical coil part and the noise generated by straight cylinder parts. In order to reduce the noise generated by the straight parts, tripping wires (T.W.) are attached on the straight parts. The diameter of tripping wire is $1 \mathrm{~mm}$, and the pitch of wires is $10 \mathrm{~mm}$. Fig. 3 shows the effect of tripping wire on the spectrum of aerodynamic noise generated by the single cylinder. A A-weighting precision sound pressure level meter is used to measure the sound levels. The position of microphone is located at an angle $\alpha=90^{\circ}$. The cylinder without the tripping wire generates the aeolian sound with a peak at $1475 \mathrm{~Hz}$. The aerodynamic sound generated by the cylinder with the tripping wire has no eminent peaks, and the overall sound pressure level $(\mathrm{OA})$ reduction of about $6 \mathrm{~dB}$ is obtained. These results show that the tripping wire is effective to reduce the aerodynamic sound generated by the cylinder. Moreover, in the case of the position of microphone is $\alpha=0^{\circ}$, the spectrum of aerodynamic noise generated by the cylinder with tripping wire nearly equals to the spectrum of BGN. The above results show that the aerodynamic noise generated by the straight cylinder parts can be avoided, when the microphone is located on $\alpha=0,180^{\circ}$ axis.

\subsection{Determination of The Microphone Position.}

The aerodynamic sound generated by the coiled wire has a unique directivity pattern. Fig.4 shows the directivity 


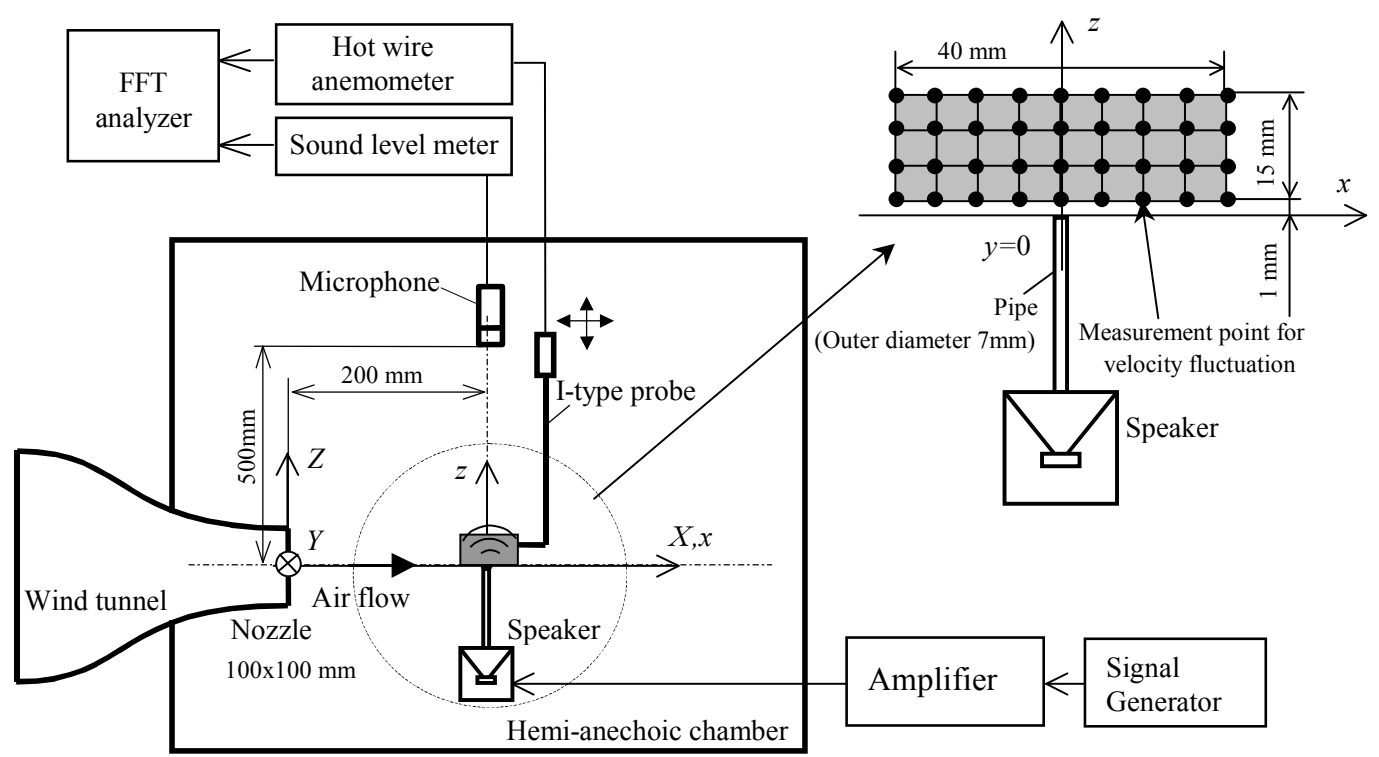

Fig.5 Experimental equipment for determination of $\psi_{v b}$

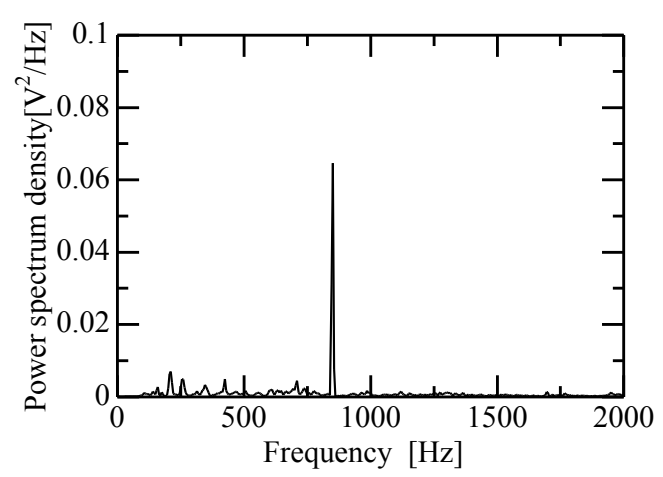

(a) Power spectrum (Sound)

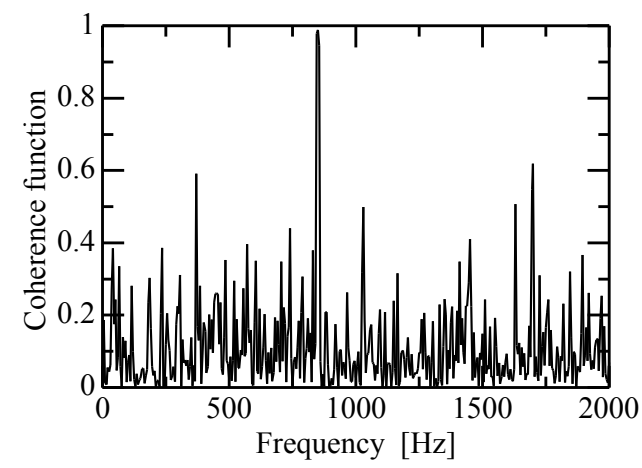

(c) Coherence function

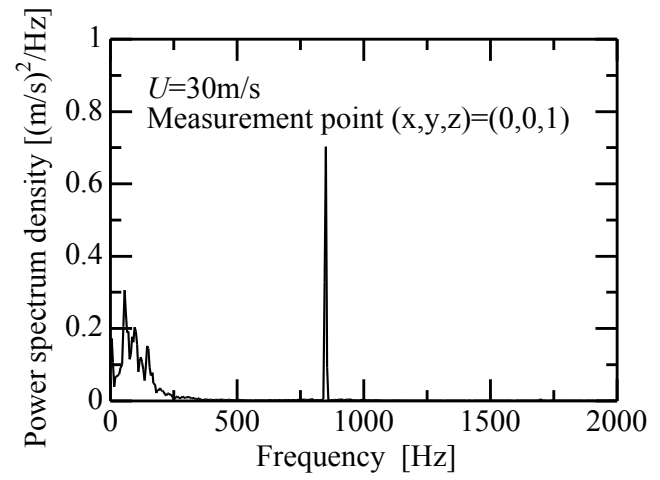

(b) Power spectrum (Velocity fluctuation)

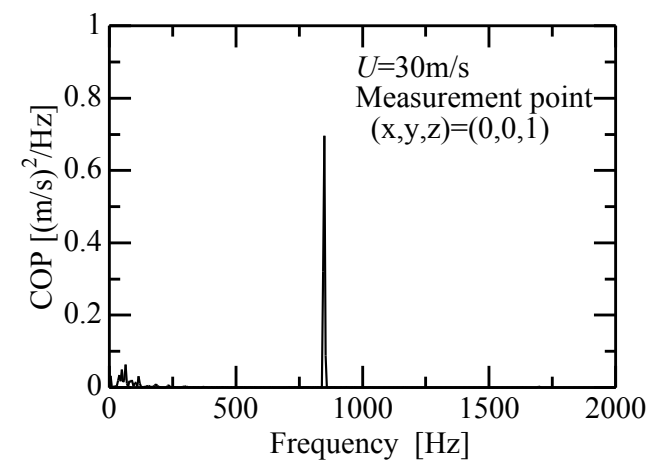

(d) Coherent output power spectrum

Fig.6 (a) Power spectrum of the sound at the microphone position $(X, Y, Z)=(200,0,500)$. (b) Power spectrum of the velocity fluctuation at the position $(x, y, z)=(0,0,1)$ (c) Coherence function. (d) Coherent output power (=Coherence function $\mathrm{x}$ Power spectrum of velocity fluctuation.) 
patterns of the coiled wire and the single cylinder having the same diameter $d=4 \mathrm{~mm}$. The plots shown in Fig. 4 indicate the normalized values by their own maximum values of overall sound level. In the case of the single cylinder, the directivity pattern has a lobe along the $\alpha=90^{\circ}$ axis, but the radiation energy of this sound source is very weak in the direction of $\alpha=0^{\circ}$ axis. In the case of coiled wire, the directivity pattern is more complicated and it has the principal lobe along the $\alpha=0^{\circ}, 180^{\circ}$ axis. In this reason, the position of the microphone is located $\alpha=0^{\circ}$.

\subsection{Measurement of The Band Over All Value of COP.}

The coherent output power (COP) is used to evaluate the correlation strength between the velocity fluctuation of the wake behind the coiled wire and the generated aerodynamic sound.

The coherence function is calculated by

$$
\gamma_{x y}^{2}(f)=\frac{\left|G_{x y}(f)\right|^{2}}{G_{x x}(f) \cdot G_{y y}(f)} \quad 0 \leq \gamma_{x y}^{2}(f) \leq 1
$$

where $G_{x x}$ and $G_{x y}$ are the power spectral density function of the sound and the sound/velocity fluctuation cross-spectral density function, respectively. $G_{y y}$ is the power spectral density function of the velocity fluctuation of the wake. $G_{x x}, G_{x y}$ are measured quantities. The coherent output power spectrum (COP) is calculated by

$$
G_{v v}(f)=\gamma_{x y}^{2}(f) \cdot G_{y y}(f)
$$

COP is the power spectrum of the velocity fluctuation that has a correlation with the aerodynamic sound generated by the coiled wire.

Furthermore, the value of COP is integrated over a specific frequency range in order to clarify the strength of the correlation between the aerodynamic sound in the specific frequency range and the velocity fluctuation of the wake behind the coiled wire. The integrated value is called as band overall value of COP. The band over all value of COP is calculated as follows,

$\psi_{v(f l-f u)}=\int_{f l}^{f u} G_{v v}(f) d f$

Here $f l$ and $f u$ are the lower frequency and the upper one of the integrated frequency range, respectively. The distribution of the normalized value of $\psi_{n v(f l-f u)}$ shows the wake region which has the strong correlation with the specific frequency range for the aerodynamic sound generated by the coiled wire. The normalized value is calculated as follows,

$$
\psi_{n v(f l-f u)}=\frac{\psi_{v(f l-f u)}}{\psi_{b v}}
$$

where $\psi_{b v}$ denotes the band overall value of COP which is obtained between a pure tone generated by a speaker and the velocity fluctuation above the sound source.

In the experiment, the microphone at a fixed position is used to measure the aerodynamic sound, and the velocity fluctuation of the wake is systematically measured at many points by the hot-wire anemometer. In order to obtain $\psi_{b v}$, a speaker with a pipe is used as the sound source in air flow. Fig. 5 shows the experimental equipments and a measurement plane. The pipe at the flow speed of $30 \mathrm{~m} / \mathrm{s}$ radiates the pure tone of $850 \mathrm{~Hz}$. The measurements are performed on a $5 \mathrm{~mm}$ grid in the $X-Z$ plane. Fig.6 shows an example of the experimental results. Fig.6(a) shows the power spectrum of the sound pressure measured by the microphone and Fig.6(b) shows the velocity fluctuation, respectively. The measurement of the velocity fluctuation is made at the position $(x, y, z)=(0,0,1)$. Fig. 6 (c) shows the coherence function between the sound pressure and the velocity fluctuation. Fig.6(d) shows COP. at the position $(x, y, z)=(0,0,1)$. As shown in Fig.6(a) and (b), the spectra have a peak at the same frequency of $850 \mathrm{~Hz}$. Thus, the coherence function has the value of 1 at $850 \mathrm{~Hz}$. Therefore, the velocity fluctuation at this measurement point and the radiated sound has strong correlation at $850 \mathrm{~Hz}$ and COP has a peak at $850 \mathrm{~Hz}$.

Fig.7 shows the distribution of $\psi_{v(850)}=G_{v v(850)}$ around the sound source in the air flow. The results show that $\psi_{v(850)}$ has the maximum value at the position $(x, y, z)=(0,0,1)$. Therefore, the location of the sound source of pure tone is 


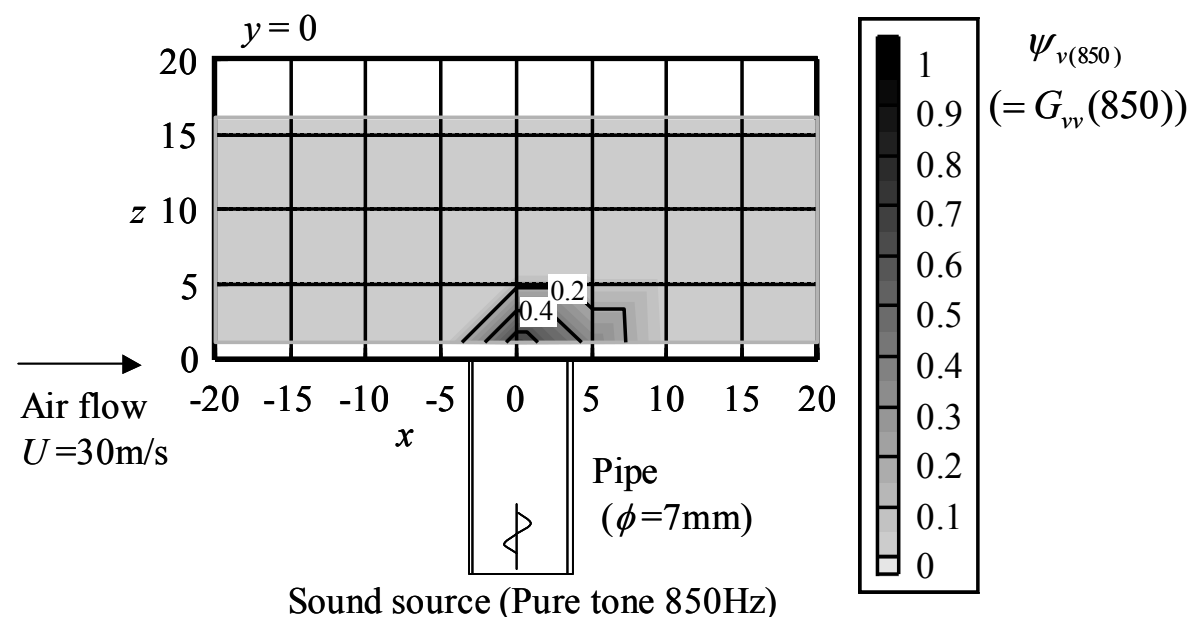

Fig.7 Distribution of a band overall value of COP around a sound source.

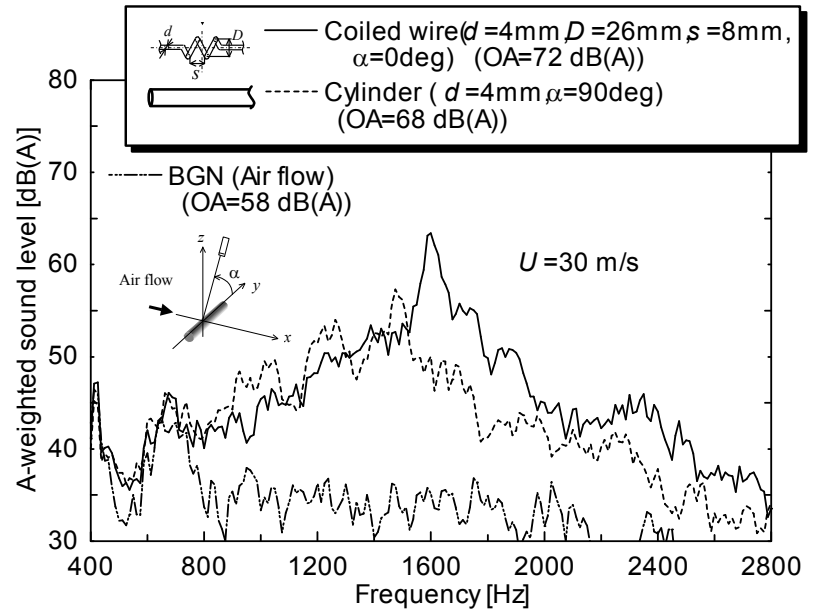

Fig. 8 Spectra of aerodynamic sounds generated by the coiled wire $(U=30 \mathrm{~m} / \mathrm{s}, d=4 \mathrm{~mm}, D=26 \mathrm{~mm}, s=8 \mathrm{~mm})$ and the single cylinder $(d=4 \mathrm{~mm})$.

determined by the results of $\psi_{v}$. In addition, we denote $\psi_{v(850)}$ measured at the position $(x, y, z)=(0,0,1)$ as $\psi_{b v}$. The coherent output power (COP) is used for the evaluation of the correlation strength between the velocity fluctuation of the wake behind the coiled wire and the generated aerodynamic sound.

\section{Experimental results}

\subsection{Characteristics of the aerodynamic sounds generated by the coiled wires}

The frequency characteristics of the aerodynamic sounds generated by the coiled wires have been examined for various values of $s$ and $D$. The example of the frequency characteristic of the aerodynamic sound generated by the coiled wire $(D=26 \mathrm{~mm}, s=8 \mathrm{~mm})$ is shown in Fig.8. The dotted line shown in the figure indicates the spectrum of aeolian sound generated by a single cylinder having the same diameter $d=4 \mathrm{~mm}$. The spectrum of the coiled wire has a prominent peak at $1600 \mathrm{~Hz}$. This peak frequency is higher than the aeolian sound generated by the single cylinder. Moreover, the overall sound level is larger than that of the single cylinder.

The effects of the coil spacing $s$ on the frequency characteristics of the aerodynamic sound generated by coiled wires are shown in Fig.9. In the case of $s=8 \mathrm{~mm}$, the frequency characteristic has a prominent peak at $1600 \mathrm{~Hz}$. 


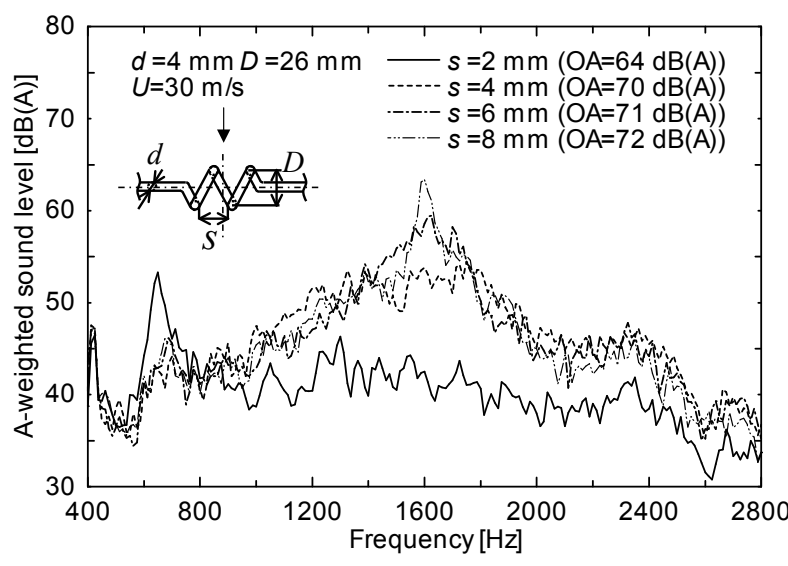

Fig.9 Effect of the spacing between coils $s$ on the aerodynamic sound generated by the coiled wire.

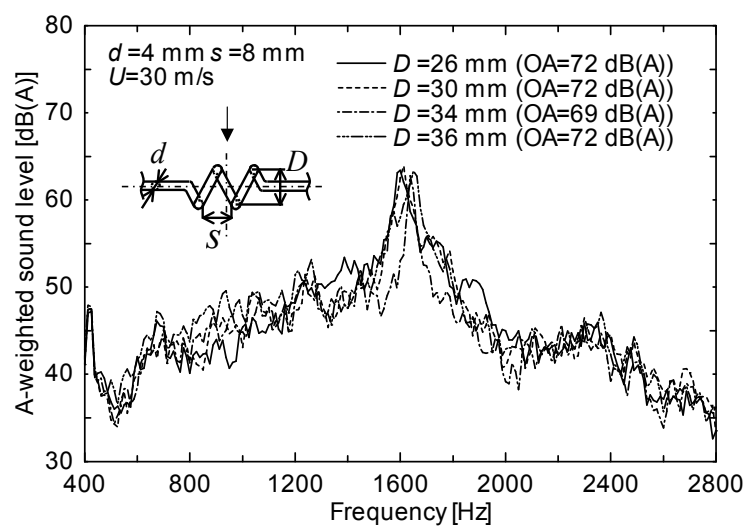

Fig.10 Effect of the outer diameter $D$ on the aerodynamic sound generated by the coiled wire.

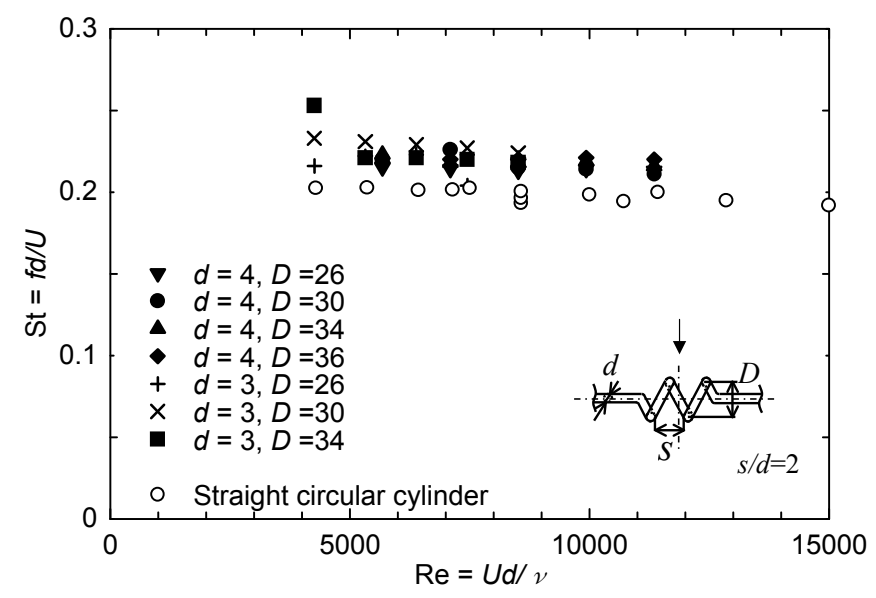

Fig.11 Relationship between Strouhal number of the coiled wire $(s / d=2)$ and Reynolds number.

However, in the case of $s=6 \mathrm{~mm}$, the peak sound level is smaller than the peak sound level in the case of $s=8 \mathrm{~mm}$. The sound level of this peak becomes weaker as $s$ decreases. In the case of $s=2 \mathrm{~mm}$, the frequency characteristic has no prominent peak at $1600 \mathrm{~Hz}$. In view of the present result, the prominent peak first appears in the spectra, when the value $s / d$ is between 1 and 1.5 .

The effects of the coil diameter $D$ on the frequency characteristics of the aerodynamic sound generated by the coiled wire are shown in Fig.10. The coil spacing $s$ is $8 \mathrm{~mm}(s / d=2)$. In all cases, the spectra have a peak around $1600 \mathrm{~Hz}$. In this condition, it can be considered that there is no effect of $D$ on the peak frequency. Moreover, the sound levels of these peaks are independent of $D$.

The Strouhal number based on $d$ is calculated. The Strouhal-Reynolds number relationship is shown in Fig.11. The Reynolds number is based on $d$. The coiled wire has a constant value of $s / d=2$. That is to say, in the case of $d=3 \mathrm{~mm}, s$ is $6 \mathrm{~mm}$. Fig. 11 shows the Strouhal number of coiled wires is about 0.22 . This value is slightly higher than the Strouhal number of the single circular cylinder. However, when the Reynolds number is between 4255 and 11347 , the corresponding Strouhal number is approximately a constant. Therefore, it is considered that this aerodynamic sound with the prominent peak can be called as an aeolian sound induced by the Karman vortex streets.

\subsection{The aerodynamic sound generated by the semicircular wire}

In order to clear the region of the vortex shedding, the semicircular wire model that is a part of the coiled wire is used. The semicircular wire is shown in Fig.12. In the case of Type-A, the arc part is located at the upstream side of air flow and in the case of Type-B, the art part is located at the downstream side of air flow. The microphone position 


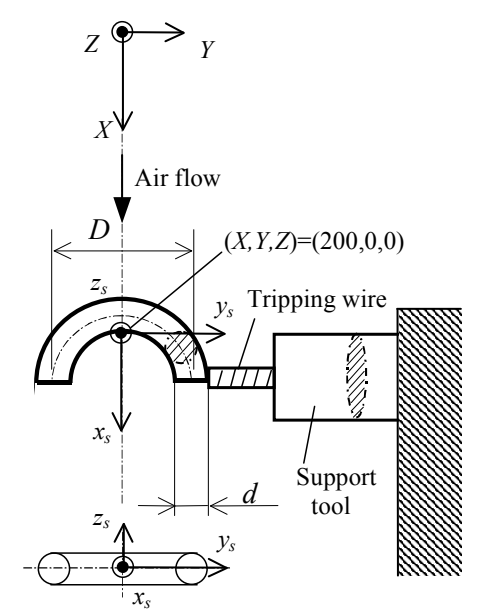

(a) Type A

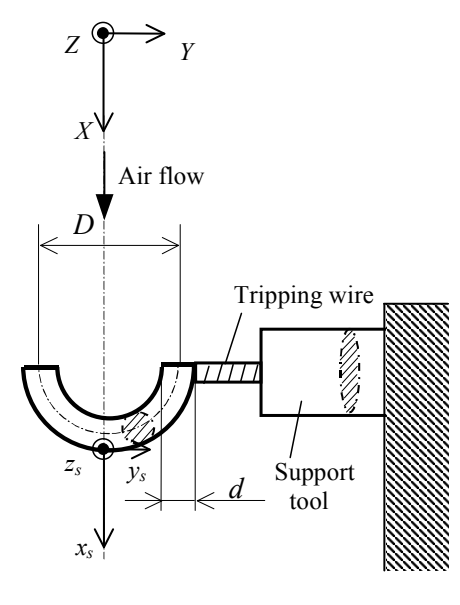

(b)Type B

Fig.12 Definition sketch of the semicircular wire. (a) Semicircular wire (Type A), (b) Semicircular wire (Type B)

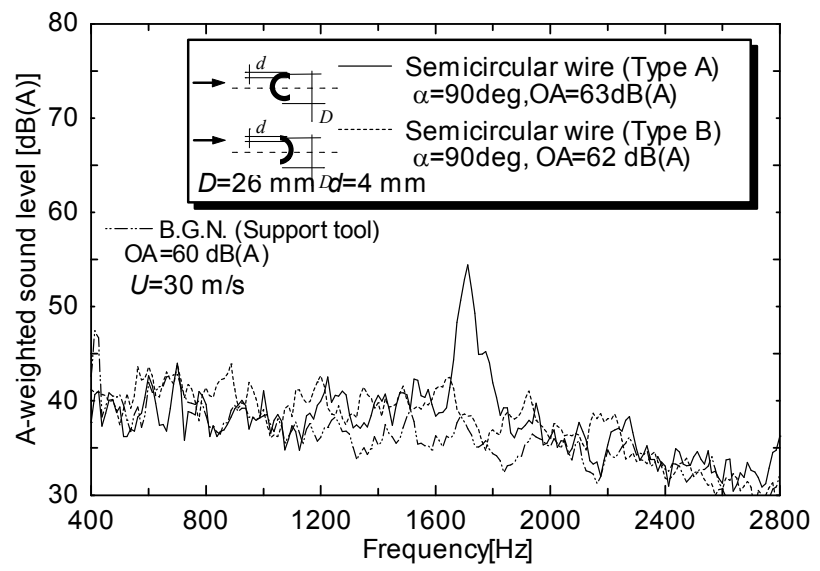

Fig.13 Spectra of aerodynamic sound generated by semicircular wires

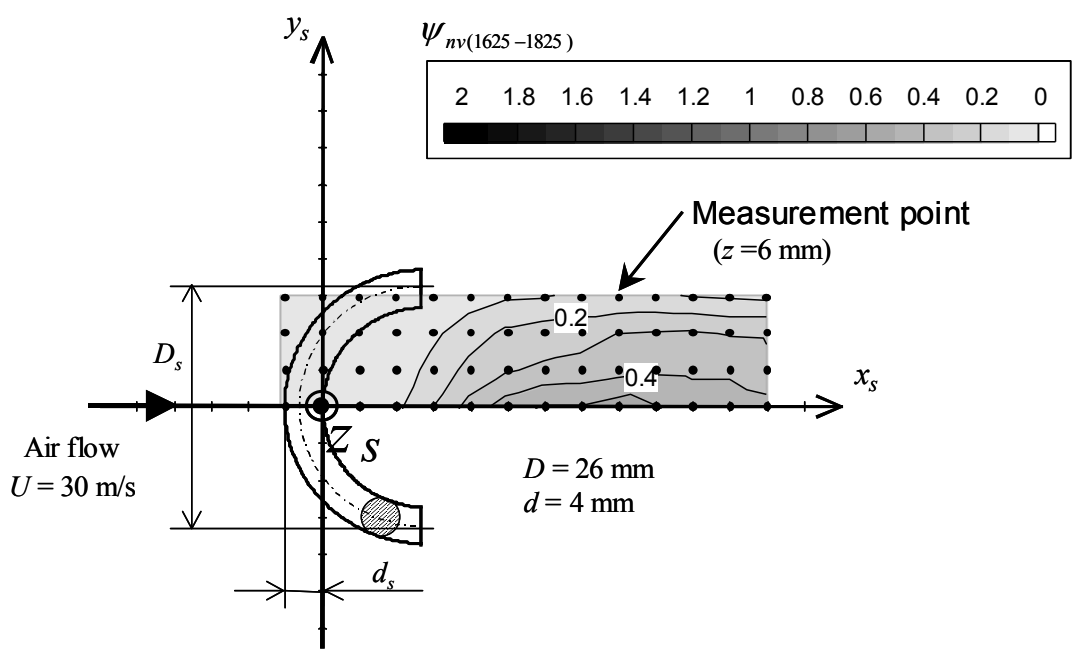

Fig.14 The distribution of $\psi_{n v(1625-1825)}$ of the Type-A semicircular wire. 


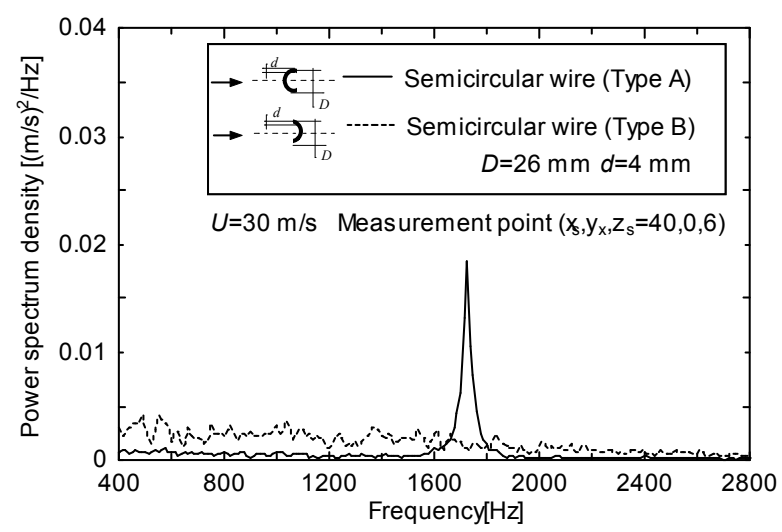

Fig.15 Power spectra of velocity fluctuation behind a semicircular wire.

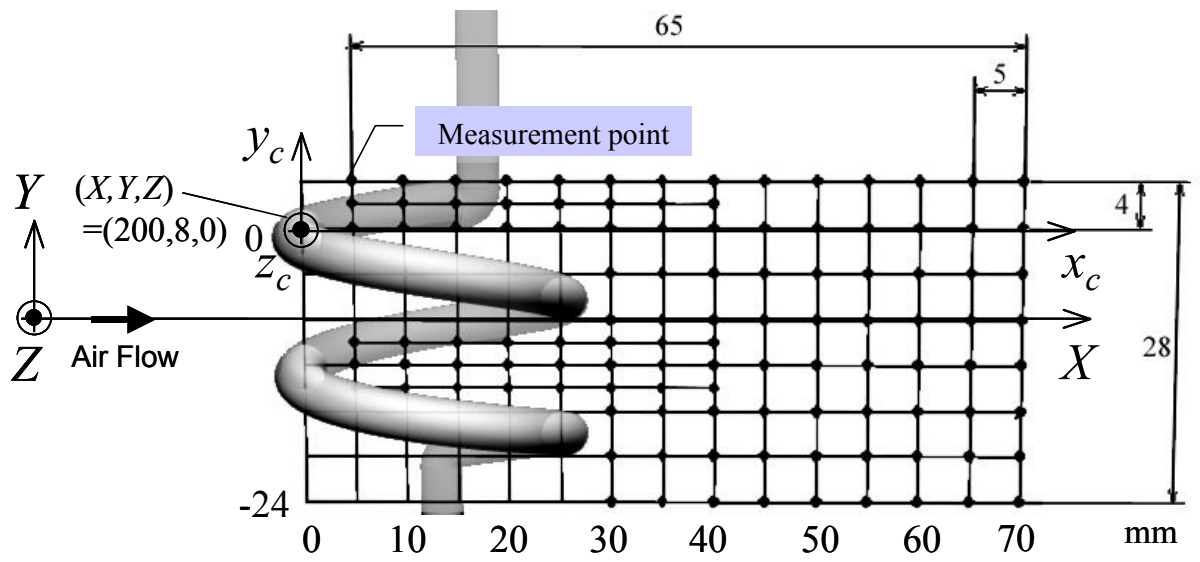

Fig.16 The measurement plane for the velocity fluctuation by the hot-wire probe.

is $\alpha=90^{\circ}$, i.e., $(X, Y, Z)=(200,0,500)$. Fig. 13 shows the spectra of the aerodynamic sounds generated by semicircular wires. The semicircular wire has the coil diameter $D=26 \mathrm{~mm}$, and the wire diameter $d=4 \mathrm{~mm}$. In the case of Type-A, the spectrum has a prominent peak at $1687 \mathrm{~Hz}$. In the case of Type-B, the peak was disappeared and the overall sound level dropped significantly.

The distribution of the band overall value of COP is shown in Fig.14. The Type A semicircular wire is used. The spectrum characteristic of aerodynamic sound generated by the Type A semicircular wire has a peak at $1687 \mathrm{~Hz}$. The $\psi_{n v(f l-f u)}$ is obtained over the frequency range of $1675-1775 \mathrm{~Hz}$. This frequency range includes the peak frequency of the aerodynamic sound generated by the Type A semicircular wire. As shown in Fig.14, the larger values of $\psi_{n v(1675-1775)}$ are distributed around the lines $x_{s}$-axis. Hence it is clear that the peak frequency of the aeolian sound and the velocity fluctuation of the wake behind the cross section on the $x_{s}$-axis of the semicircular wire (the center part of the semicircular wire) have strong correlation in the region along the $x_{s}$-axis. It is considered that the flow that breaks away from the upper and the lower sides of the cross section of the semicircular wire is fluctuating at the same frequency of the aeolian sound. As the result, it has become clear that the aeolian sound source is generated in the wake region behind the center part of the semicircular wire. Fig. 15 shows the comparison with the spectrum of velocity fluctuation behind the Type A semicircular wire and that of Type-B. Both measurement positions are $\left(x_{s}, y_{s}\right.$, $\left.z_{s}\right)=(40,0,6)$, respectively. The spectrum curve of Type A has a prominent peak at $1687 \mathrm{~Hz}$ which is the same peak frequency of the sound spectrum. But the spectrum curve of the Type B has no prominent peaks and it is similar to a spectrum curve of a random noise. Therefore, the wake of Type A has a periodic fluctuation, but the wake of Type B is a turbulent flow. In the case of coiled wire, it is found that the aeolian sound is generated by the wake of the arc part which is located at the upstream side of air flow. 


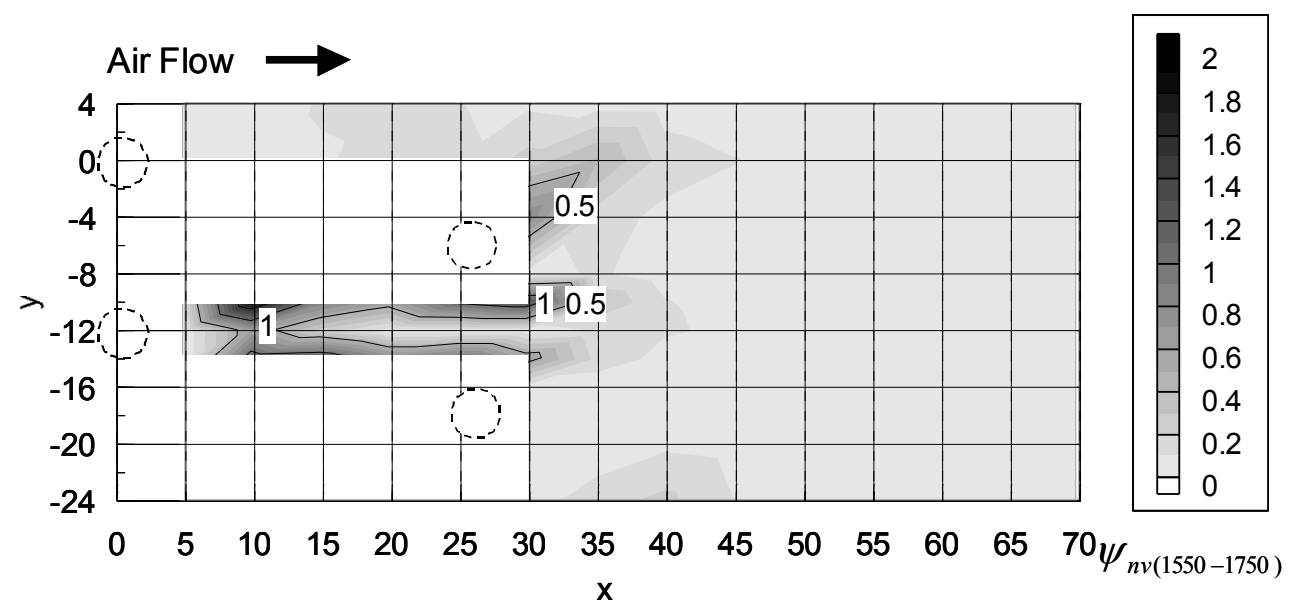

(a) 2-D view

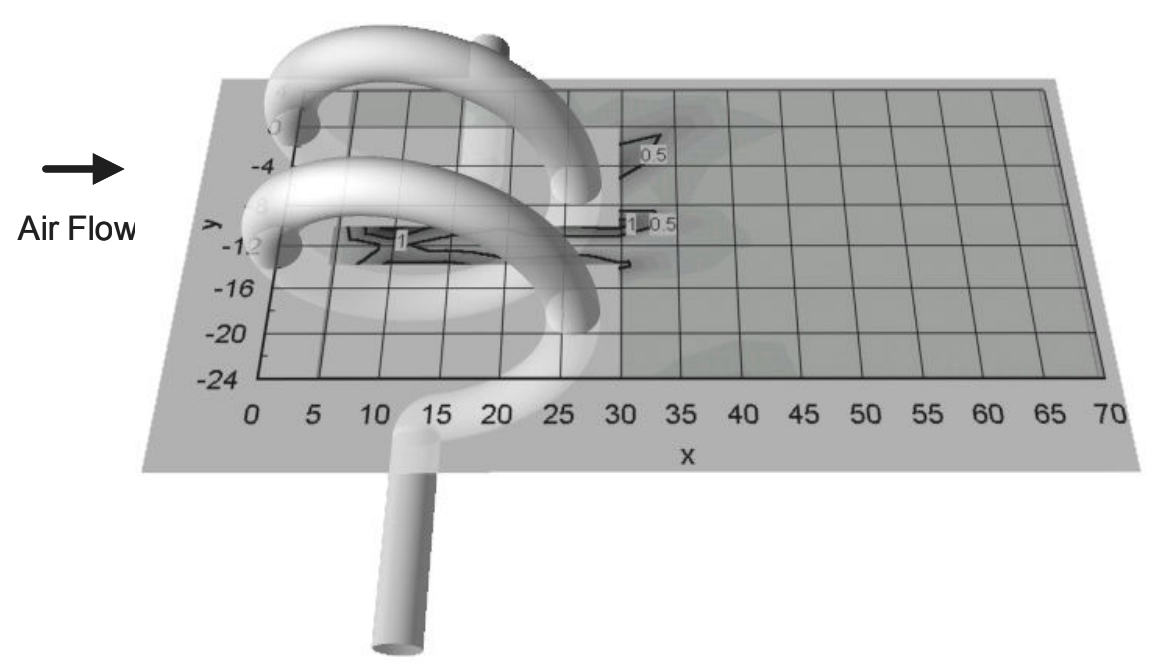

(b) 3-D view

Fig.17 Distribution of the band overall value of COP $\left(\psi_{n v(1550-1750)}\right)$ of the coiled wire.

\subsection{The distribution of $\psi_{n v}$ of the coiled wire}

In order to clear the location of sound sources of the coiled wire, the distribution of the band overall of COP has been measured. The measurement plane for the distribution of $\psi_{n v}$ is defined as Fig.16. The measurement plane is a $X-Y$ plane located at $Z=0$ (containing the axis of the coil). On basis of above experimental results, it is observed that this plane is containing a larger value of $\psi_{n v}$.

Fig. 17 shows the distribution of $\psi_{n v}$ for a coiled wire of having coil diameter $D$, wire diameter $d$ and coil spacing $s$ in the frequency range between 1550-1750Hz. Fig.17 (a) and Fig.17 (b) show 2-dimensional view and 3-dimensional view of the distribution of $\psi_{n v}$, respectively. The spectrum characteristic of aerodynamic sound generated by this coiled wire is shown in Fig.8. The peak frequency of the aeolian sound is $1600 \mathrm{~Hz}$. The larger value of $\psi_{n v(1550-1750)}$ are distributed around the lines $y=-10,-14 \mathrm{~mm}$. But in the wake regions of downstream sides of coiled wire $(x>30 \mathrm{~mm})$, the values of $\psi_{n v(1550-1750)}$ are small.

Therefore, the aerodynamic sound generated by the coiled wire is dominated by the vortex shedding at the upstream side of the coil. And, we found that the vortex shedding from the upstream side of the coil interacts to downstream side of it, and then, the vortex is collapsed by the wake of downstream side of the coil. Figs.18(a) (d) are examples of power spectrum of velocity fluctuation in the wake of the downstream side of the coil. The measurements are performed at the position of $x=25 \mathrm{~mm}$ to $40 \mathrm{~mm}$ for an interval of $5 \mathrm{~mm}$ on the line, $y=-10 \mathrm{~mm}$. 


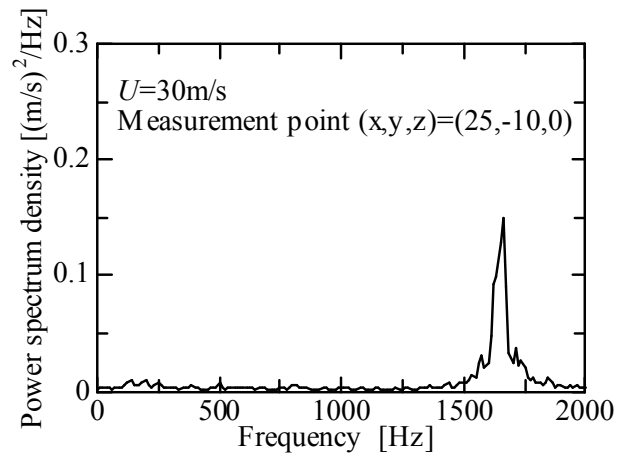

(a) Power spectrum (Velocity fluctuation) at the point $(x, y, z)=(25,-10,0)$

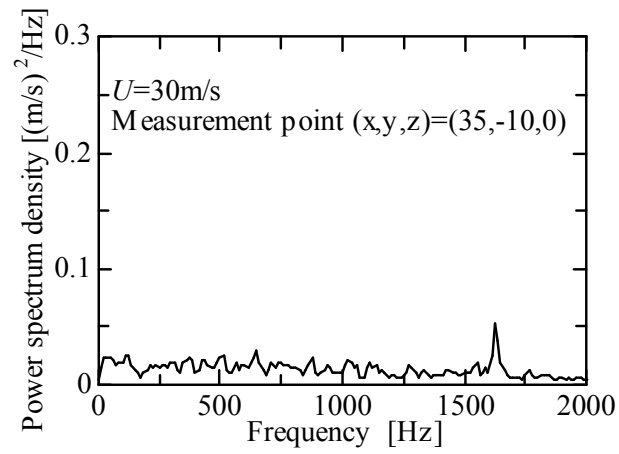

(c) Power spectrum (Velocity fluctuation) at the point $(\mathrm{x}, \mathrm{y}, \mathrm{z})=(35,-10,0)$

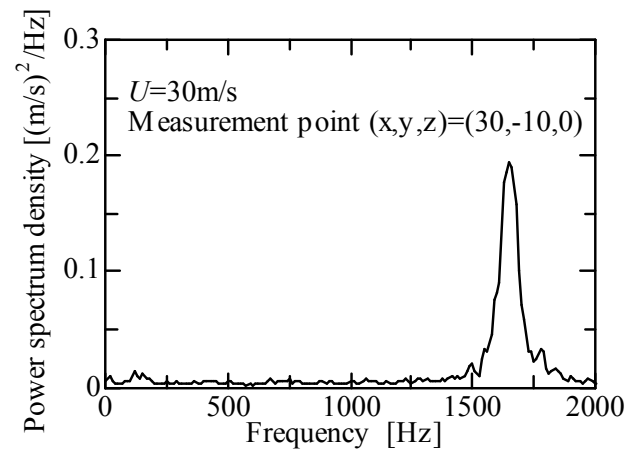

(b) Power spectrum (Velocity fluctuation) at the point $(\mathrm{x}, \mathrm{y}, \mathrm{z})=(30,-10,0)$

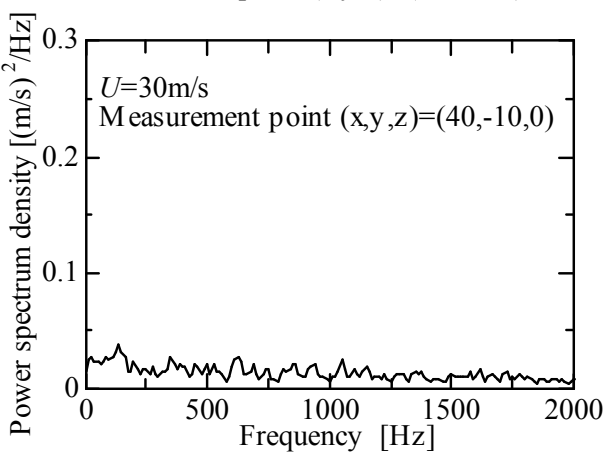

(d) Power spectrum (Velocity fluctuation) at the point $(x, y, z)=(40,-10,0)$

Fig.18 Power spectrum of velocity fluctuation. (a) The measurement point $(x, y, z)=(25,-10,0)$, (b) $(30,-10,0)$, (c) $(35,-10,0), \quad(d)(40,-10,0)$

As the results shown in Fig.18 (a), the power spectrum of velocity fluctuation has the peak at $1600 \mathrm{~Hz}$ that corresponds with the peak frequency of the sound. The peak becomes weaker when the measurement point is $x=35$ (Fig.18(c)). Fig.18 (d) shows the power spectrum density for the measurement position $(40,-10,0)$, there is no peak at $1600 \mathrm{~Hz}$ and this power spectrum has larger value in a broad frequency range. At this measurement position the velocity fluctuation becomes disorder. From these results we confirmed that the vortex collapsed in the wake behind downstream sides of the coiled wire.

The configuration of the centre portions of upstream and downstream coil wires resembles a staggered cylinder row. Sumner et al. (Sumner, D. et al (2000)) identified the flow pattern boundaries for two staggered cylinders. According to the map of Sumner et al., the flow pattern of the case of this coiled wire is located on the boundary line between "synchronized vortex shedding" (SVS) and "vortex impingement"(VI), if Reynolds number was 850-1900. In the case of this coiled wire (Fig.17), Reynolds number is about 8571. It is difficult to explain about the vortex behavior only using COP data. We could not confirm the vortex impinges or not impinge in this case. In the literature the Strouhal number of this coiled wire's case is about 0.2 . But in our case, the Strouhal number is 0.22 , i.e. it is slightly higher than that of the literature. This reason is not clear at the present moment, and extended research works are necessary to achieve the goal.

\section{Conclusions}

In this paper, the characteristics of the aerodynamic sounds generated by coiled wires have been examined. Moreover, concerning the correlation between the aerodynamic sound and the velocity fluctuation of the wake behind the coiled wire is researched by using the method of measuring $\psi_{n v(f l-f u)}$ (the band overall value of coherent output power). The results obtained may be summarized as follows. 
1. When $s / d$ is greater than 1 , the spectrum of the aerodynamic sound generated by the coiled wire has a prominent peak.

2. The directivity pattern of the aerodynamic sound source due to the coiled wire differs from the aeolian sound source due to a single cylinder.

3. Using the method of measuring $\psi_{n v(f l-f u)}$, it is possible to clarify the distribution of the strong correlation between the velocity fluctuation in the wake and the aerodynamic sound generated by the coiled wire.

4. The aerodynamic sound generated by the coiled wire is dominated by the vortex shedding from the upstream side arc part of the coiled wire.

\section{Acknowledgments}

This research has been supported by a research grant from Research Fellowships of the Japan Society for the Promotion of Science for Young Scientists.

\section{References}

A.Iida, T.Otaguro, Y. Yakano, and H.Fujita, 1998. Visualization of aerodynamic sound source in a turbulent wake. Proc. INTER-NOISE 98, Paper No.38.

Bendat, J. S. and Piersol, A. G., 1980. Engineering Applications of Correlation and Spectral analysis, John Wiley \& Sons

Sumner, D., Price, S. J., Païdoussis, M. P., 2000. Flow-pattern identification for two staggered circular cylinders in cross-flow. J. Fluid Mech., vol.411, pp263-303

H. Fujita, J. Shiraishi, T. Kurita, Y. Maruta, S. Yamada, 1996. Acoustical and aerodynamic characteristics of end plate materials for two-dimensional models in low noise wind tunnel experiments. Proc. INTER-NOISE 96, pp.1227-1230.

H. Fujita, S. Yamada, Y. Maruta, and H. Maki, 1997. Control of aerodynamic noise generated from two-dimensional models. Proc. INTER-NOISE 97, pp.371-374.

O. Mochizuki, M. Kiya, T. Suzuki, T. Arai, 1994. Vortex-Shedding Sound Generated by Two Circular Cylinders Arranged in Tandem. Trans. Jpn. Soc. Mech. Eng. (in Japanese), 60-578, pp.1-7. 